\title{
Pilot experimental study on amniotic epithelial mesenchymal cell transplantation in natural occurring tendinopathy in horses. Ultrasonographic and histological comparison
}

\author{
Aurelio Muttini ${ }^{1}$ \\ Valentina Russo ${ }^{1}$ \\ Elisabetta Rossi² \\ Mauro Mattioli' \\ Barbara Barboni ${ }^{1}$ \\ Umberto Tosi ${ }^{1}$ \\ Nicola Maffulli ${ }^{3}$ \\ Luca Valbonetti 1 \\ Michele Abate ${ }^{4}$
}

${ }^{1}$ Department of Comparative Biomedical Sciences, University of Teramo, Italy

2 Viral Vaccines Institute, Serum and Diagnostics, Abruzzo and Molise Region Experimental Animal Health Care Institute "G. Caporale" (IZSAM), Teramo, Italy

${ }^{3}$ Department of Physical and Rehabilitation Medicine, University of Salerno, Azienda Ospedaliera San Giovanni di Dio e Ruggi d'Aragona, Salerno, Italy; Centre for Sports and Exercise Medicine, Queen Mary University of London, Barts and The London School of Medicine and Dentistry, Mile End Hospital, London, UK

4 Department of Medicine and Science of Aging, University G. d'Annunzio, Chieti-Pescara, Chieti Scalo, Italy

Corresponding author:

Michele Abate

Department of Medicine and Sciences of Aging, University "G. d 'Annunzio" Chieti-Pescara

Via dei Vestini 31

66013 Chieti Scalo, Italy

E-mail:m.abate@unich.it

\section{Summary}

Background: amnion-derived stem cells are considered a promising alternative source for tendon tissue regeneration.

Purpose: aims of this paper were to illustrate the ultrasound and histological outcomes following the treatment of acute and chronic superficial digital flexor tendon spontaneous lesions in horses with ovine amniotic epithelial cells xenotransplantation. Methods: six adult horses suffering from unilateral acute ( 4 cases) and chronic ( 2 cases) tendinopathy (clinical and ultrasound diagnosis) were enrolled. At baseline, ovine amniotic epithelial cells were grafted, in sterile conditions and under ultrasound control, into the most damaged area. Ultrasound controls were performed at $30,60,90,120$, 150 and 180 days after cells implantation; after horse euthanasia (180 days) tendon samples were collected and submitted to histological examination (cellularity, extracellular matrix fiber organization, blood vessels).

Results: at baseline, in the acute cases, the ultrasound exam showed a focal, dis-homogeneous, hypo-echoic area into the superficial digital flexor tendon, with loss of the normal fibrillar pattern, while in the chronic cases the damaged tendon area appeared thickened and completely hyperechoic. At the final follow-up tendon echotexture was more regular, the cross-sectional area similar to the contra-lateral limb, and the collagen fibers were oriented in parallel to the longitudinal axis of the tendon both in the acute and chronic cases, suggesting a positive healing response.

These findings were supported by the histological analyses which showed an almost complete restoration of normal tendon architecture with an optimal alignment of tendon fibers.

Conclusions: the present pilot study supports the hypothesis that amniotic epithelial cells are provided of an excellent healing potential and shows a very good correlation between the ultrasound findings and the histologic features.

KEY WORDS: amniotic epithelial cell, histology, tendon, ultrasound, xenotrasplantation.

\section{Introduction}

Adult tendons do not heal thorough a regenerative process ${ }^{1}$, but with the formation of a fibrotic scar, which is characterized by an abnormal collagen fiber alignment and weakened biomechanical properties $^{2,3}$. For this reason, re-injury rate following superficial digital flexor tendon (SDFT) lesions in horses is very high and can reach $43 \% 4,5$. Because the poor outcome is likely to be related to the limited regenerative capacity of tenocytes ${ }^{6}$, an increasing interest has been devoted to stem cells based therapies ${ }^{7}$. At this purpose, Hertel first reported positive results with an implant of whole bone marrow (BM) into suspensory ligament lesions in horses ${ }^{8}$. After this seminal work, the autologous implantation of in vitro cultured BM-derived mesenchymal stem cells (BM- 
MSCs) has been widely used in the treatment of equine tendon and ligament injuries ${ }^{9}$, showing significant improvements in the structure and biomechanical properties of the tendons. However, some practical and biological concerns related to BM-MSCs auto-transplantation have been raised, including the morbidity for the donor site, the delay between the $\mathrm{BM}$ collection and the grafting procedure, and the difficulties in cells standardization ${ }^{10,11}$. For these reasons, many authors have recently investigated alternative sources for MSCs, including adipose and tendon tissues ${ }^{12}$. Amnion-derived stem cells (AECs) are considered a promising alternative of stem cells to be used in tendon tissue engineering ${ }^{13}$, since they conjugate a remarkable plasticity with safety properties $^{14-16}$. In a recent study it has been demonstrated that, when co-cultured with equine tenocytes, ovine AECs (oAECs) can differentiate in tenocytes and that, after transplantation into equine SDFT spontaneous acute tendon lesions, they allow very good clinical results and the restoration of a quite normal ultrasound (US) pattern of tendon fibers orientation $^{17}$. However, the clinical nature of the study has precluded a proper standardization of the US evaluation of the healing process and a comparison between US and histological features.

Moreover, it must be added that all the clinical studies reported in literature refer to the use of MSCs in acute lesions, and that the treatment of chronic cases has never been attempted. Therefore, the aim of the present research was twofold: first, to study with a standardized protocol the US and histological outcomes following the treatment of acute SDFT spontaneous lesions with oAECs xenotransplantation; second, to illustrate, for the first time, the efficacy of this treatment in the spontaneous chronic SDFT lesions.

\section{Materials and methods}

\section{Animals}

This study was approved by the local Ethical Committee (PROT. 48/2011/CEISA/COM 07/07/2011) and was conducted in compliance with the Italian Animal Welfare guidelines following the ethical standard of the Muscles, Ligaments and Tendons Journal ${ }^{18}$. Six adult horses suffering from acute and chronic SDFT tendinopathy were included in the study. The inclusion criteria were the following: retirement from activity; no treatment in the previous 15 days (in acute cases) and 4 months (in chronic cases) before the beginning of the study; lesion located in the mid metacarpal region where the tendon is not surrounded by the sheath at US evaluation.

\section{Ultrasonography}

Before enrollment, horses underwent an US and Power Doppler (PD) evaluation of the affected tendon, using a high-resolution, multi-frequency (6-15
$\mathrm{MHz}$ ) linear array transducer. The assessment was performed by the same operator (AM) using both longitudinal and transverse scans, with horses standing and bearing weight on the examined limb. Both forelimbs were examined in order to exclude the involvement of the contra-lateral one.

The US criteria adopted for the diagnosis of tendinopathy were the following:

1) the presence of dishomogeneous hypo- or hyperechoic thickening, diffuse or focal, of the mid metacarpal region of the tendon, associated with loss of the normal fibrillar pattern and/or irregularity of the tendon margins and/or increased cross-sectional area, was interpreted as a sign of degeneration; 2) the presence of peritendinopathy was diagnosed when a patchy thickening of the paratenon, associated or not with irregularities of tendon margins, was found; and 3) neovascularization was estimated by means of PD and graded as (0), (1), (2), (3), (4), according to the appearance of vessels inside the region of interest. In detail (0) is no Doppler activity; (1), one or two tiny foci; (2), up to $50 \%$ colour inside the region of interest; (3), $50-90 \%$ colour inside the region of interest; (4), $90-100 \%$ colour inside the region of interest ${ }^{19}$. To avoid artifacts, sensitivity was optimized for low flow, and the gain was set just below the noise level. Further sonographic controls were performed at 30,60 , $90,120,150$ days after oAECs implantation. The last US and PD examination was performed at 180 days (horses euthanasia).

\section{oAECs isolation, culture, characterization and storage}

The preparation of oAECs has been previously described $^{17}$. Briefly, placentas were collected from slaughtered sheep at 3 months of pregnancy. The cells were obtained from the isolated amniotic epithelial layer after enzymatic digestion (0,25\% trypsin-EDTA, Sigma Chemical Co. ${ }^{\circledR}$, St. Louis, MO, USA). After culture, oAECs were screened by flow cytometry for the major surface molecules cluster of differentiation and for intracellular stem cell markers. The oAECs ability to differentiate into osteogenic ${ }^{20}$ and tenogenic ${ }^{6}$ lineages was tested prior to their implantation as previously reported. In particular, tenocytes were isolated after in vitro incubation of SDFT tendon explants collected from slaughtered adult horses. Tenogenic differentiation was monitored after 28 days of co-culture by analyzing the ability of oAECs cultured alone (CTR) or exposed to primary tenocytes (co-culture) to down regulate cytokeratin 8 (epithelial marker [immunohistochemical analysis]), to generate three dimensional tendon-like structures (stereomicroscope and inverted microscope), to express collagen type I (COL1) and tendon-ligament related genes (RT-PCR technique). The oAECs with a stable proliferation index (expressed as mean doubling time which was about 15-20 h in all fetuses analyzed during the first three passages of in vitro expansion), a conserved expression pattern and the ability to un- 
dergo in vitro tenogenic cell lineage differentiation, were stored in vials of $2.5 \times 106$ by cryopreservation in liquid nitrogen.

\section{oAECs implantation}

The procedure was performed as previously described ${ }^{17}$, with the horses standing and sedated using detomidine $10 \mu \mathrm{g} / \mathrm{kg}$ (Domosedan ${ }^{\circledR}$ - Pfizer, Italy) and butorphanol $20 \mu \mathrm{g} / \mathrm{kg}$ (Dolorex ${ }^{\circledR}$, Intervet, Italy). Before implantation the intended pool of cells was defrosted. Briefly, after regional analgesia, cell injection was always performed with the limb bearing weight. After aseptic preparation, the tip of a needle (20G X 1.5 inch) was guided, under US control, into the defect where a core lesion (acute) was present, or in the area where US showed the most severe upset of tendon echo-texture in chronic cases. A total of $7 x$ 106 oAECs in $500 \mu \mathrm{l}$ of $\alpha$ MEM was grafted into the tendon. Limbs were then bandaged and ice packs were daily applied for a few days. Horses were rested in box for 7 days before commencing a rehabilitation period equal for all animals and consisting in daily hand-walking and trotting.

\section{Tendon analysis and histological examination}

Horses were euthanized after 180 days post-cell implantation by an overdose of thiopental (Pentothal Sodium $^{\circledR}$, Intervet) and embutramide (Tanax ${ }^{\circledR}$, Intervet). Samples were submitted to histological examination.

The explanted tendons were transversally cut at least $5 \mathrm{~mm}$ from the injured area. A portion of the contralateral healthy tendon (same level) was also collected for comparison. The specimens were immediately cryopreserved; longitudinal sections were cryosectioned at $7 \mu \mathrm{m}$-thickness, collected on glass slides, and stained with haematoxylin-eosin (H\&E) to collect information on tissue micro-architecture and on collagen fibers reorganization within the injured tendon. In brief, for H\&E stain sections were brought to water and the nuclei stained with an alum hematoxylin (Sig$\mathrm{ma}^{\circledR}$, St. Louis, MO) for 5 minutes. Then, slides were immersed into tap water for 5 minutes. The cytoplasm was contrasted with Eosin (Sigma ${ }^{\circledR}$, St. Louis, MO) for 5 minutes, and then rinsed well with distilled water. Finally the sections were mounted with an aqueous medium (Sigma ${ }^{\circledR}$, St. Louis, MO).
Cellularity, extracellular matrix fiber organization, and blood vessels were used to describe tendon healing process. Finally, all stained sections were analyzed with the Axioscop2 plus microscope (Zeiss, Oberkochen, Germany).

\section{Results}

\section{oAECs isolation, culture, characterization and storage}

Accordingly to previous studies $6,13,17$ in vitro expanded oAECs showed a constant proliferative ability, a conserved phenotype and stable expression profile of stemness markers. Differentiation into tenocytes was also regularly documented.

\section{Clinical data}

Four horses affected from unilateral acute SDFT tendinopathy and two horses with chronic SDFT tendinopathy were enrolled. Typical signs of acute (heat, swelling, pain on palpation, lameness) or chronic (profile deformity, thickness and lameness) tendinopathy were present in all cases. Demographic (age, sex, discipline) and clinical data (lameness score [evaluated according to American Association of Equine Practitioners (AAEP) scoring system ${ }^{21}$ ], time of onset of symptomatology, mean injury duration) are reported in Table 1. The transplantation procedure was well tolerated by all horses that did not exhibit any discomfort or adverse effects. No antibiotics or other drugs were administered at any time. The clinical outcome was characterized by a progressive reduction of lameness (during walking and trotting), which was no more detectable on an average of three weeks post oAECs implantation.

\section{Ultrasound features}

At baseline, US signs of tendon lesions were observed in four right (three acute and one chronic) and two left (one acute and one chronic lesions) thoracic limbs, respectively; the contra-lateral limb of all horses did not show any US damage.

In the four acute lesions, US examination showed a focal, dishomogeneous, hypo-echoic area into the

Table 1. Athletic performance's signalment of the horses combined with the clinical evaluation of lesion.

\begin{tabular}{llllll}
\hline Case number & Aptitude & Sex & Age (years) & Lesion type & Lameness scoring * \\
\hline 1 & Dressage & Gelding & 4 & Acute & 2 \\
2 & Racing Flat & Stallion & 3 & Acute & 1 \\
3 & Racing Flat & Mare & 4 & Acute & 2 \\
4 & Racing Flat & Gelding & 5 & Acute & 2 \\
5 & Racing Flat & Mare & 7 & Chronic & 2 \\
6 & Eventing & Gelding & 7 & Chronic & 2 \\
\hline
\end{tabular}

* According to AAEP scoring system 


\section{A. Muttini et al.}

SDF tendon, with loss of the normal fibrillar pattern. In the two tendons with chronic damage a longitudinal hyper-echoic area with a similar pattern, located in the medial portion of the tendon, was observed (Fig. 1). Neovessels were never detected at PD evaluation. Thirty days after transplantation in all horses with acute damage the affected areas, compared with baseline, appeared widened (increased cross-sectional area), and the sonographic changes were still present; progressively, from the day 60 to the day 90 , the cross-sectional area, was found comparably reduced, however showing an irregular contour, and the echotexture was more homogeneous. Finally, at
120,150 and 180 days after the treatment, the echotexture was more regular, the cross-sectional area similar to the contra-lateral limb, and the collagen fibers were oriented in parallel to the longitudinal axis of the tendon (Fig. 1).

In the two chronic lesions, at 30 days after transplantation, the damaged areas appeared less hyperechoic but without any change in the cross-sectional area (Fig. 2); a progressive reduction in the echogenicity and in the cross-sectional area was then observed after 60, 90, 120 and 150 days. At the final follow up (180 days), tendons resumed a quite normal appearance (Fig. 2). During the whole duration of

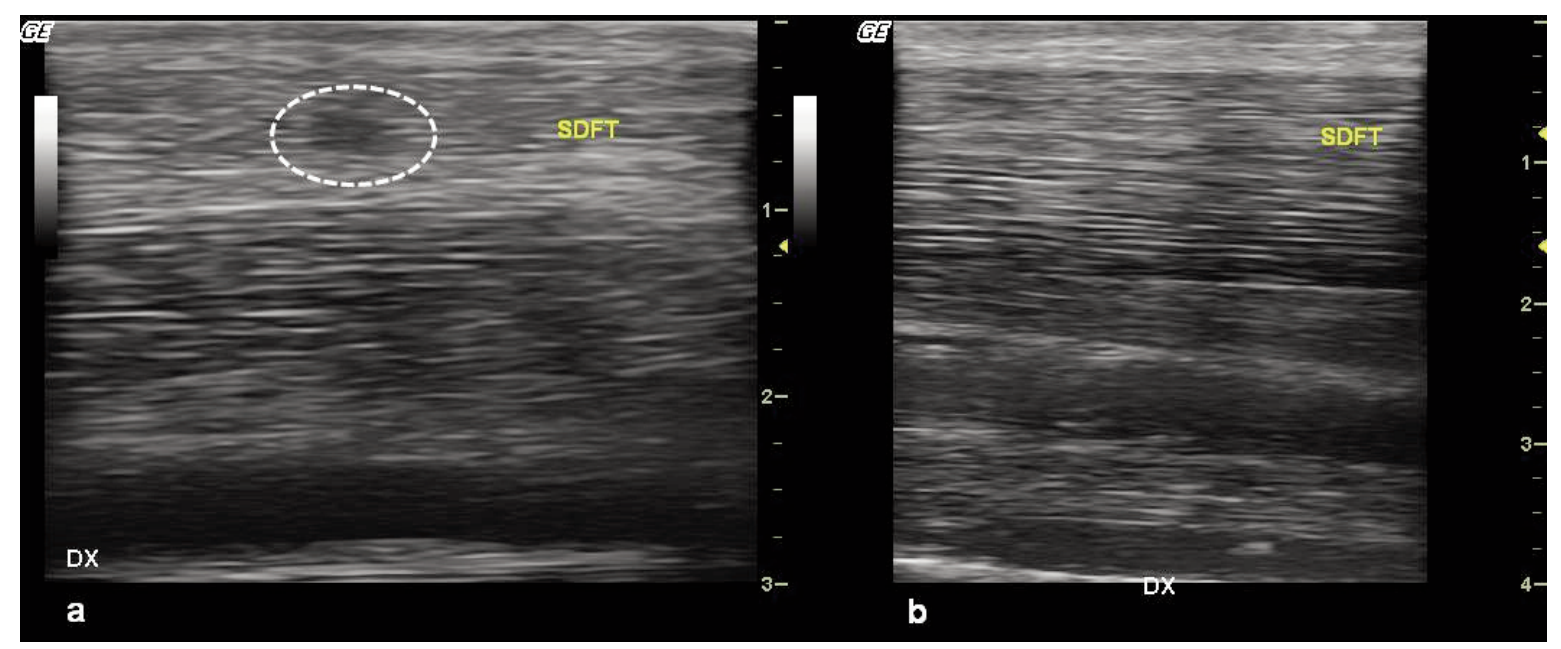

Figure 1. Ultrasound features of an acute lesion.

At baseline (panel a), the presence of a focal, dishomogeneous, hypo-echoic area (dotted circle) into the SDF tendon, with loss of the normal fibrillar pattern, is observed (core lesion).

At the final follow-up (180 days after treatment, panel b) the tendon seems to be healed at US exam: indeed, the echotexture is more regular, the cross-sectional area similar to the contra-lateral limb, and the collagen fibers are oriented in parallel to the longitudinal axis of the tendon.

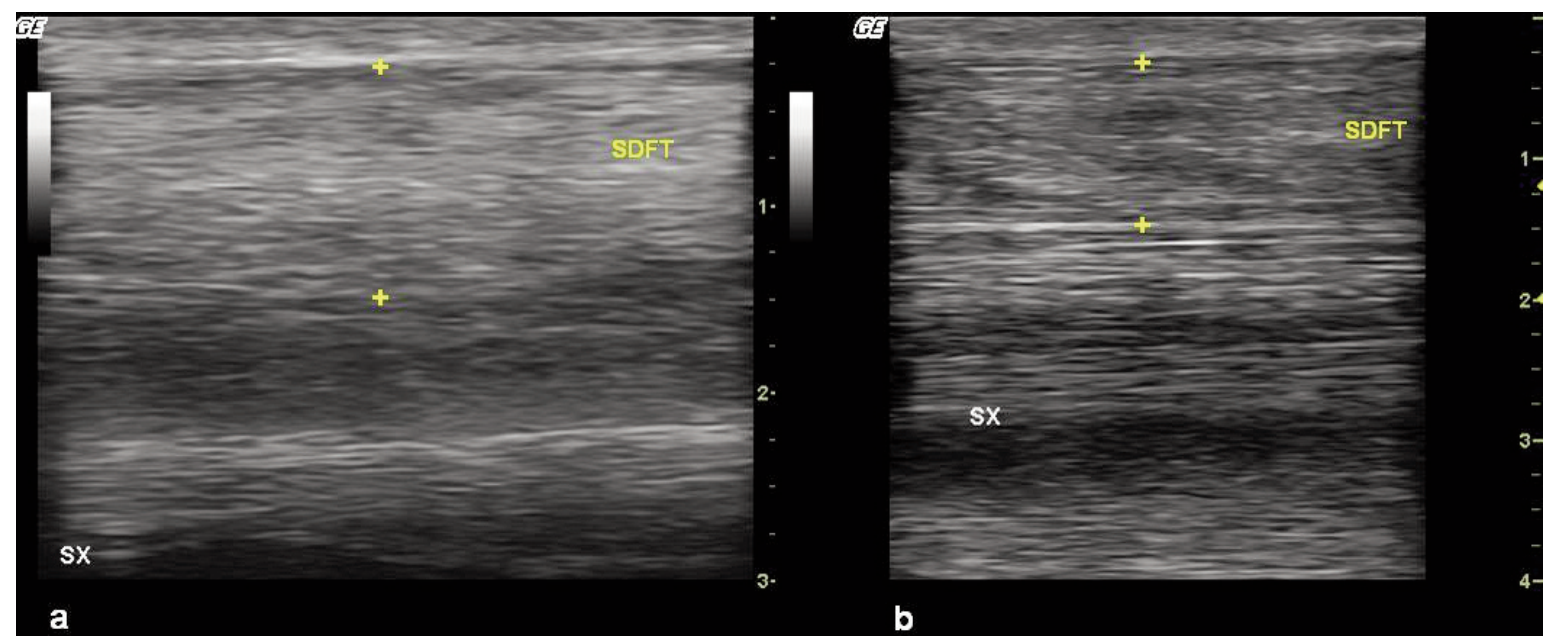

Figure 2. Ultrasound features of a chronic lesion.

At baseline (panel a), the tendon damaged area into the SDFT appears thickened and completely hyper-echoic, with loss of the normal fibrillar pattern.

At the final follow up (180 days after treatment, panel b), a progressive reduction in echogenicity and in cross-sectional area is observed, with the tendon resuming a quite normal appearance. 
the study neovascularization was never detected at the PD exam.

\section{Tendon histology}

The histological analyses of the oAECs treated-tendons showed an advanced healing process that involved the whole injured area both in acute and chronic lesions (Fig. 3). Tendon and endotenon connective tissues were properly and similarly restored in both cases. The interface between the two tissue types was readily defined. The proper tendon tissue consisted of large groups of collagen fibrils lying in parallel alignment, with tenocytes, characterized by a typical fusiform shape, among them. In detail, a higher concentration of fusiform cells was present within the lesion site, compared to the healthy tissue. These cells were surrounded by an abundant extracellular matrix organized in parallel fibers oriented along the longitudinal axis of the tendon. The extracellular matrix within the regenerated site closely resembled a healthy tissue with a similar eosin staining density. Endotenon layers, composed of loose connective tissue and rounded cells, were running within the proper tendon tissue. Blood vessels, contained within the endotenon, did not show any difference between regenerated and control healthy tendons and were aligned along the longitudinal axis of the tendon as in healthy ones.

At the end of the co-cultural period (28 days) some bundle structures became three dimensional, while CTR oAECs reached a confluent monolayer. Immunohistochemistry analysis showed that all fusiform cells lost their positivity for the epithelial marker Cytokeratin
8, while strongly increased their content in COL1 protein, differently from CTR oAECs. The co-culture significantly changed oAECs gene profiles increasing COL1 and SCX and decreasing COL3 mRNA content. The intracellular mRNA levels of all the three tendon related genes became similar to those recorded in ovine tendons analyzed as control tissues.

\section{Discussion}

Many experimental and clinical studies published in recent years have shown the potential therapeutic benefit and safety of MSCs in tendon repair ${ }^{9,22}$. These cells can be obtained by different sources (autologous and heterologous), but their use is hampered by technical difficulties. On the other hand, AECs can be easily collected, expanded in vitro with a constant proliferative ability, a conserved phenotype, and a stable expression profile of stemness markers $^{12}$. Their differentiation into tenocytes is also regularly documented ${ }^{12}$. Moreover, these cells can be stored thanks to cryopreservation and used for allogeneic transplantation in veterinary medicine. Previous experimental studies, in mechanically-induced acute tendon injuries in large animals species (sheep), have shown their good tolerance by the host, due to their low immunologic potential, and their efficacy in regenerating tendon ${ }^{6,17}$. Very good clinical results have been also obtained after transplantation into equine SDF acute tendinopathies, with restoration of a quite normal US pattern of tendon fibers orientation ${ }^{17}$. However the clinical nature of this study has precluded a proper standardization of the US
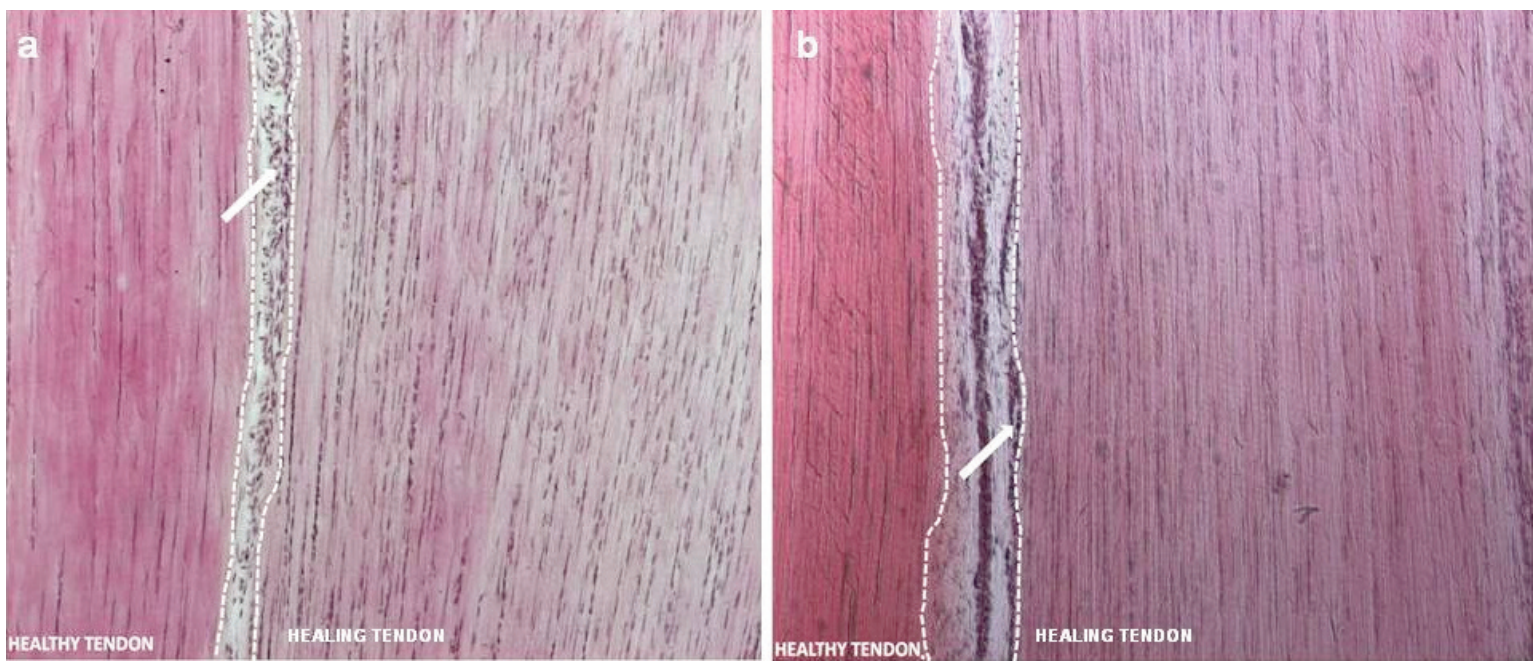

Figure 3. Morphological analysis of advanced healing process in acute and chronic SDFT lesions. H\&E stain micrographs (100X magnification) of SDFT tissue sections at 180 days post treatment with oAECs.

In the micrographs, the healing and the healthy tissue is on the right and on the left of dotted lines respectively, and the endotenon with blood vessels in the area between them. The images show that, both in acute (panel a) and chronic (panel b) lesions, tendon and endotenon connective tissue are properly restored. Collagen fibrils are parallelly aligned, and tenocytes are characterized by a typical fusiform shape. Blood vessels (arrows) are visible exclusively within the endotenon.

In the regenerating area a higher nuclei concentration and a lower density of the extracellular matrix fibers in comparison with the healthy tendon are evident. 
evaluation of the healing process and a comparison between US and histological features.

The present study enlarges our knowledge on acute tendinopathies, not only confirming the excellent clinical outcome after implantation, as previously reported $^{17}$, but also showing a very good correlation between the US findings and the histologic features of tendon samples, collected 180 days after implantation. Indeed, the US evaluation has shown a decrease of the cross-sectional area at the level of maximal injured zone and the recovery of a normal alignment pattern of tendon fibers, similar to that observed in the contra-lateral healthy tendon. The early widening of the core lesions is considered normal during the inflammatory phase and is due to fluid accumulation during the digestion of disrupted fibers by proteolytic enzymes and their removal by phagocytosis ${ }^{23}$. Accordingly, at histological evaluation, it has been observed an almost complete restoration of normal tendon architecture with an optimal alignment of tendon fibers and blood vessels, although a high cellularity still present suggests that the regenerative process was not completely concluded.

The new finding reported in the present study, first in literature, is the demonstration of efficacy also in chronic spontaneous tendinopathies. Again, this assumption is demonstrated not only by the clinical improvement, but also by the normalization of US architecture and the corresponding histological analysis that showed an almost normal structure of the tissue. Surprisingly tendon histology in chronic lesions after treatment was completely superimposable to that of the acute ones. Therefore, it may be concluded that the xenotransplantation of ovine AECs into horses is able to normalize the mechanical and structural parameters of treated tendons, irrespective of the stage of the lesions.

It is well known from histologic observations that the tendon damage develops in a continuum in different stages: the acute lesions are characterized by tissue necrosis, oedema, and infiltrates of inflammatory cells, with overproduction of inflammatory cytokines; subsequently extracellular matrix is formed and organized in fibrils, which, however, do not reproduce the normal tendon architecture, and evolves in a fibrotic scar. Several growth factors are necessary to fuel and support the tendon regeneration. Tenocytes play a pivotal role, not only by means of the growth factors they produce, but also soliciting a proper and physiologic alignment of the newly formed fibers ${ }^{24}$. The equivalent results, as improvement in clinical symptoms, US and histologic features, observed in the present study, support the hypothesis that stem cells, differentiating into tenocytes, are equally useful and induce a regenerative process both in the acute and chronic stages of tendinopathy, irrespective of the biochemical and histologic environment.

It is noteworthy that equine and human tendinopathies have many similarities in their pathophysiology25,26, since equine SDFT recapitulates the main biomechanical and histologic characteristics of human Achilles tendon. However, further comparative studies are needed to establish an equivalence also in spontaneous pathologic situations, before considering the use of AECs for translational perspectives.

Some limitations of the present study must be acknowledged. First, it is mandatory to increase the number of cases (mainly of chronic tendinopathy) before definitely stating that the treatment with oAECs can enhance tendon regeneration. Second, it could be useful to know the number of cells to be injected in relation to the size of the lesion for the best results. Third, it will be necessary to evaluate whether the clinical improvement is also present in conditions of stressful exercise.

In conclusion, the use of heterologous AECs is very promising because of the good results, the easy availability and the low immunogenic potential. However, larger studies and a comparison with control groups treated with stem cells of different source are needed to establish their therapeutic potential in the treatment of tendinopathies.

"This work has not been published previously, it is not under consideration for publication elsewhere, its publication is approved by all Authors and by the responsible authorities where the work was carried out".

\section{Declaration of conflicting interests}

The authors declared no potential conflicts of interests with respect to the authorship and/or publication of this article.

\section{Funding}

The authors received no financial support for the research and/or authorship of this article.

\section{References}

1. Favata M, Beredjiklian PK, Zgonis MH, et al. Regenerative properties of fetal sheep tendon are not adversely affected by transplantation into an adult environment. J Orthop Res. 2006;24(11):2124-2132.

2. Sharma P, Maffulli N. Biology of tendon injury: healing, modeling and remodeling. J Musculoskelet Neuronal Interact. 2006;6(2):181-190.

3. Sharma P, Maffulli N. Tendon injury and tendinopathy: healing and repair. J Bone Joint Surg Am. 2005;87(1):187-202.

4. Dyson SJ. Treatment of superficial digital flexor tendinitis: a comparison of conservative management, sodium hyaluronate, and glycosaminoglycan polysulfate, in Proceedings. 43rd Annu Conv Am Assoc Equine Pract. 1997;297-300.

5. Dyson SJ. Medical management of superficial digital flexor tendonitis: a comparative study in 219 horses (1992-2000). Equine Vet J. 2004;36(5):415-419.

6. Barboni B, Russo V, Curini V, et al. Achilles tendon regeneration can be improved by amniotic epithelial cell allotransplantation. Cell Transplant. 2012;21(11):2377-2395. 
7. Kryger GS, Chong AK, Costa M, Pham H, Bates SJ, Chang J. A comparison of tenocytes and mesenchymal stem cells for use in flexor tendon tissue engineering. J Hand Surg Am. 2007;32(5):597-605.

8. Hertel D. Enhanced suspensory ligament healing in 100 horses by stem cells and other bone marrow components. Proc Am Assoc equine Practice. 2001;47:319.

9. Smith RKW, Werling NJ, Dakin SG, Atam R, Goodship AE, Dudhia J. Beneficial effects of autologous bone marrow-derived mesenchymal stem cells in naturally occurring tendinopathy. PLoS One. 2013;8(9):e75697.

10. Guest DJ, Smith MRW, Allen WR. Equine embryonic stem-like cells and mesenchymal stromal cells have different survival rates and migration patterns following their injection into damaged superficial digital flexor tendon. Equine Vet J. 2010;42 (7):636-642.

11. Paris DB, Stout TA. Equine embryos and embryonic stem cells: Defining reliable markers of pluripotency. Theriogenology. 2010;74(4):516-524.

12. Muttini A, SaliniV, Valbonetti L, Abate M. Stem cell therapy of tendinopathies. Muscles Ligaments Tendons Journal. 2012;2(3):187-192.

13. Barboni B, Curini V, Russo V, et al. Indirect co-culture with tendons or tendocytes can program amniotic epithelial cells towards stepwise tenogenic differentiation. PLoS One. 2012;7 (2): $\mathrm{e} 30974$.

14. De Coppi P, Bartsch G Jr, Siddiqui MM, et al. Isolation of amniotic stem cell lines with potential for therapy. Nat Biotechnol. 2007;25(1):100-106.

15. Parolini O, Soncini M, Evangelista M, Schmidt D. Amniotic membrane and amniotic fluid-derived cells: Potential tools for regenerative medicine? Regen Med. 2009;4(2):275-291.

16. Antonucci I, Stuppia L, Kaneko Y, et al. Amniotic fluid as a rich source of mesenchymal stromal cells for transplantation therapy. Cell Transplant. 2011;20(6):789-795.

17. Muttini A, Valbonetti L, Abate M, et al. Ovine amniotic epithelial cells: In vitro characterization and transplantation into equine superficial digital flexor tendon spontaneous defects. Res Vet Sci. 2013;94(1):158-169.

18. Padulo J, Oliva F, Frizziero A, Maffulli N. Muscles, Ligaments and Tendons Journal. Basic principles and recommendations in clinical and field science research. MLTJ. 2013;4:250-252.

19. Boesen MI, Torp-Pedersen S, Koenig MJ, et al. Ultrasound guided electrocoagulation in patients with chronic non-insertional Achilles tendinopathy: a pilot study. Br J Sports Med. 2006;40(9):761-766.

20. Mattioli M, Gloria A, Turriani M, et al. Stemness characteristics and osteogenic potential of sheep amniotic epithelial cells. Cell Biol Int. 2012;36(1):7-19.

21. Dwyer A. Science-in-brief: clinical highlights from the American Association of Equine Practitioners 59th Annual Convention and Equine Veterinary Journal Supplement 45. Equine Vet J. 2014;46(3):259-261.

22. Lange-Consiglio A, Rossi D, Tassan D, Perego R, Cremonesi F, Parolini O. Conditioned medium from horse amniotic membrane derived multipotent progenitor cells: immunomodulatory activity in vitro and first clinical application in tendon and ligament injuries in vivo. Stem Cells Dev. 2013;22(22):3015-3024.

23. Bosch G, Lameris MC, van den Belt AJM, Barneveld A, van Weeren PR. The propagation of induced tendon lesions in the equine superficial digital flexor tendon: an ex vivo study. Equine Vet J. 2010;42(5):407-411.

24. Leversedge FJ, Zelouf D, Williams C, Gelberman RH, Seller JG III. Flexor tendon grafting to the hand: an assessment of the intrasynovial donor tendon-A preliminary single-cohort study. J Hand Surg Am. 2000;25(4):721-730.

25. Smith RKW. Mesenchymal stem cell therapy for equine tendinopathy. Disabil Rehabil. 2008;30(20-22):1752-1758.

26. Lui PP, Maffulli N, Rolf C, Smth RK. What are validated animal models for tendinopathy? Scand J Med Sci Sports. 2011; 21(1):3-17. 